\title{
Statistical analysis of linear spatial holes estimators in cognitive radio
}

\author{
Mohammad Kazemi ${ }^{*}$, Mehrdad Ardebilipour and Behrad Mahboobi
}

\begin{abstract}
One of key features of cognitive radio (CR) networks is environment awareness which is having knowledge of such parameters as spatial holes. This information is employed to exploit the spatial resources more efficiently and limit the interference to the primary users to an admissible level. In order to evaluate the performance of a spatial holes estimation algorithm, statistical characteristics of its estimation error must be compared to a benchmark such as Cramer-Rao lower bound (CRLB). In this article, the performance of cognitive RSS-WLS algorithm which is an important linear spatial hole estimation algorithm in CR systems has been analyzed by obtaining the closed form expression for mean and covariance of its estimation. Then its performance is compared with CRLB and it is shown that cognitive RSS-WLS estimator is asymptotically efficient.
\end{abstract}

\section{Introduction}

Increasing demand of radio bandwidth in recent years plus inefficient use of licensed bands has encouraged the researchers to devise a mechanism to allocate the radio bandwidth in a more efficient way. One of the most promising ideas to overcome this problem was cognitive radio (CR) which was first introduced by Mitola [1]. A CR secondary network tries to coexist with a primary network. The primary network is a network that owns a licensed radio band and the secondary network is a network which wants to use the same band without causing any harmful interference to the primary network.

One of key features of CR networks is environment awareness which is having knowledge of such parameters as spatial holes. By employing spatial holes like location and power information of primary network users, secondary users can reduce their interference to the primary network by efficient utilization of this information in beamforming and power control algorithms. To choose the best estimator when implementing the cognitive system, or to utilize the hybrid estimators, the error of each spatial holes estimation as a performance measure, should be analyzed by comparing them with a common benchmark like Cramer-Rao lower bound (CRLB).

In this article, it is assumed that there is no cooperation or known signaling between secondary and primary users.

\footnotetext{
* Correspondence: mohammadkaazemi@yahoo.com

Faculty of Electrical and Computer Engineering, Khaje Nasir Toosi University
} of Technology, Tehran, Iran

(C) 2012 Kazemi et al; licensee Springer. This is an Open Access article distributed under the terms of the Creative Commons Attribution License (http://creativecommons.org/licenses/by/2.0), which permits unrestricted use, distribution, and reproduction in any medium, provided the original work is properly cited.
Hence, algorithms which need cooperation between primary and secondary networks cannot be used. For example, because of the need of synchronization between primary and secondary networks, time-based algorithms like time of arrival (TOA) algorithm cannot be used in the $\mathrm{CR}$ case. The problem which appears in angle of arrival (AOA) algorithms is that although they have no problem estimating the locations of the primary users, they are unable to estimate their transmission powers. Hence among all of the common location estimation algorithms only the received signal strength (RSS) algorithms can be used for simultaneous estimation of locations and powers of the primary users in a CR network. To estimate the location of the primary user, RSS algorithms measure and process the received power of the primary user's signal at the secondary users' receivers.

There have not been a lot of works in the field of simultaneous estimation of location and power, especially in CR networks. In [2], a linear RSS algorithm based on weighted least square (WLS) method is introduced and analyzed which is called RSS-WLS. In WLS as a generalization of LS method, the matrix of coefficients is multiplied by a weighting matrix to let the system of linear equations be solved using LS method. In [3], a spatial holes estimation algorithm, namely RoTPE, is proposed based on RSS-WLS algorithm which can estimate primary users' powers and locations simultaneously. This algorithm rearranges RSSWLS algorithm to add the capability of estimating the power of the primary user in a CR network setup. In [4],

\section{是 Springer}


Kazemi et al. introduce cognitive RSS-WLS which improves [3] by taking input noise and error of path loss exponent estimation into account in addition to shadowing. Although $[3,4]$ propose an RSS-WLS based simultaneous location and power estimation algorithm for CR setup, they do not do any exact or approximate analytical performance analysis.

In this article, the performance of cognitive RSS-WLS approach of [4] in an AWGN channel has been analyzed by obtaining the closed form expression for mean and covariance of its estimation error and comparing it with CRLB. in this article, first CRLB of RSS-based algorithms for CR setup in an AWGN channel, is obtained. Then, the closed form expression for mean and covariance of estimation error of cognitive RSS-WLS algorithm is calculated. At the end, based on the results of the previous sections, an analytical performance evaluation of cognitive RSS-WLS algorithm is done and it is shown that cognitive RSS-WLS estimator is asymptotically efficient.

\section{Cramer-Rao lower bound (CRLB)}

Cramer-Rao lower bound (CRLB) expresses a lower bound on the variance of any unbiased estimator of a deterministic parameter. RSS-based algorithms try to estimate location and power of a primary user using RSS measurements of the secondary users, based on a propagation model which depends on the communication channel. In an AWGN channel in CR network, the RSS at $i$ th secondary user, $R S S_{i}$, is formulated by the following propagation model,

$$
R S S_{i}=K_{i} \frac{p}{d_{i}^{\alpha}}+n
$$

where $d_{i}$ is the distance between the primary user and the $i$ th secondary user, and $p$ is the transmission power of primary user which due to lack of cooperation between primary and secondary networks, is assumed unknown. In Equation (1), $\alpha$ is the path loss exponent, and $K_{i}$ is all other factors that affect the received signal power including the antennas gain and height and the signal carrier frequency.

Let the real location of the primary user be $[x, y]$ and the coordinate of the $i$ th secondary user be $\left[x_{i}, y_{i}\right], i=1$, $2, \ldots, M$, where $M$ is the total number of the secondary receiver users. The distance between the primary user and the $i$ th secondary user, $d_{i}$, can be calculated by the following equation:

$$
d_{i}=\sqrt{\left(x-x_{i}\right)^{2}+\left(y-y_{i}\right)^{2}}, \quad i=1,2, \ldots, M .
$$

RSS measurement error, $n$, can be generated by channel noise or any other random perturbations to the measurement system and in AWGN channel it is assumed to be a zero mean Gaussian random variable with sufficiently small variance compared to the received signal power, i.e., $n \sim N\left(0, \sigma_{n}^{2}\right)$, hence,

$$
\operatorname{RSS}_{i} \sim N\left(\frac{K_{i} p}{d_{i}^{\alpha}}, \quad \sigma_{n}^{2}\right)
$$

Assuming the RSS measurements in different secondary users are independent, using (2), joint probability density function (pdf) of RSS measurements of all secondary users is as follows,

$$
\begin{aligned}
& f_{R S S}(R S S ; \theta)=\prod_{i=1}^{M} f_{R S S_{i}}\left(R S S_{i} ; \theta\right) \\
& =\frac{1}{\left(2 \pi \sigma_{n}^{2}\right)^{\frac{M}{2}}} \exp \left(-\frac{1}{2 \sigma_{n}^{2}} \sum_{i=1}^{M}\left(R S S_{i}-\frac{K_{i} p}{d_{i}^{\alpha}}\right)^{2}\right)
\end{aligned}
$$

where $\boldsymbol{\theta}=(x, y, p)$ represent the set of unknown parameters. Using (3), natural logarithm of joint pdf of RSS measurements, $L(R S S ; \theta)$, can be written as,

$$
\begin{aligned}
& L(R S S ; \theta) \triangleq \ln \left(f_{R S S}(R S S ; \theta)\right) \\
& \quad=-\frac{M}{2} \ln \left(2 \pi \sigma_{n}^{2}\right)-\frac{1}{2 \sigma_{n}^{2}} \sum_{i=1}^{M}\left(R S S_{i}-\frac{K_{i} p}{d_{i}^{\alpha}}\right)^{2}
\end{aligned}
$$

Now that all the prerequisites have been calculated, CRLB can be obtained using the following equation [5],

$$
C R L B=J^{-1},
$$

where $J$ is the Fisher information matrix (FIM) whose elements can be calculated using the following equation,

$$
J_{i j}=E\left[\frac{\partial L(R S S ; \theta)}{\partial \theta_{i}} \frac{\partial L(R S S ; \theta)}{\partial \theta_{j}}\right] .
$$

In order to obtain CRLB, using (7) we first calculate the elements of $J$. Since the number of parameters to be estimated in RSS-based location and power estimation algorithms is 3, 9 elements need to be calculated. According to (7), $J$ is symmetric; therefore the number of elements to be calculated reduces to 6. Using (2), these 6 elements are calculated as follows,

$$
\begin{aligned}
& J_{11}=E\left[\left(\frac{\partial L(R S S ; \theta)}{\partial x}\right)^{2}\right] \\
&=\frac{\alpha^{2} p^{2}}{\sigma_{n}^{4}} E\left[\left(\sum_{i=1}^{M} \frac{1}{d_{i}^{\alpha+2}} K_{i}\left(x-x_{i}\right)\left(R S S_{i}-\frac{K_{i} p}{d_{i}^{*}}\right)\right)^{2}\right] \\
& \begin{array}{c}
\text { RSS } S_{i} \text { are } \\
\text { independent }
\end{array} \\
& \stackrel{\alpha^{2} p^{2}}{\sigma_{n}^{4}} \sum_{i=1}^{M}\left(\frac{1}{d_{i}^{(2 \alpha+2)}} K_{i}^{2}\left(x-x_{i}\right)^{2} E\left[\left(R S S_{i}-\frac{K_{i} p}{d_{i}^{*}}\right)^{2}\right]\right) \\
&=\frac{\alpha^{2} p^{2}}{\sigma_{n}^{2}} \sum_{i=1}^{M} \frac{K_{i}^{2}\left(x-x_{i}\right)^{2}}{d_{i}^{2(\alpha+2)}}
\end{aligned}
$$




$$
\begin{aligned}
& J_{33}=E\left[\left(\frac{\partial L(R S S ; \theta)}{\partial p}\right)^{2}\right]=\frac{1}{\sigma_{n}^{4}} E\left[\left(\sum_{i=1}^{M} \frac{K_{i}}{d_{i}^{\alpha}}\left(R S S_{i}-\frac{K_{i} p}{d_{i}^{\alpha}}\right)\right)^{2}\right] \\
& \stackrel{\substack{R S S_{i} \text { s are } \\
\text { independent }}}{=} \frac{1}{\sigma_{n}^{4}} \sum_{i=1}^{M}\left(\frac{K_{i}^{2}}{d_{i}^{2 \alpha}} E\left[\left(R S S_{i}-\frac{K_{i} p}{d_{i}^{\alpha}}\right)^{2}\right]\right)=\frac{1}{\sigma_{n}^{2}} \sum_{i=1}^{M} \frac{K_{i}^{2}}{d_{i}^{2 \alpha}} \\
& J_{12}=J_{21}=E\left[\left(\frac{\partial L(R S S ; \theta)}{\partial x}\right)\left(\frac{\partial L(R S S ; \theta)}{\partial y}\right)\right] \\
& =\frac{\alpha^{2} p^{2}}{\sigma_{n}^{4}} E\left[\sum_{i=1}^{M}\left(\frac{K_{i}\left(x-x_{i}\right)}{d_{i}^{\alpha+2}}\left(R S S_{i}-\frac{K_{i} p}{d_{i}^{\alpha}}\right)\right) \sum_{i=1}^{M}\left(\frac{K_{i}\left(\gamma-y_{i}\right)}{d_{i}^{\alpha+2}}\left(R^{\alpha} S S_{i}-\frac{K_{i} p}{d_{i}^{\alpha}}\right)\right)\right] \\
& \stackrel{\substack{R S S_{i} \text { s are } \\
\text { independent }}}{=} \frac{\alpha^{2} p^{2}}{\sigma_{n}^{4}} \sum_{i=1}^{M}\left(\frac{K_{i}^{2}\left(x-x_{i}\right)\left(y-\gamma_{i}\right)}{d_{i}^{2(\alpha+2)}} E\left[\left(R S S_{i}-\frac{K_{i} p}{d_{i}^{\alpha}}\right)^{2}\right]\right) \\
& =\frac{\alpha^{2} p^{2}}{\sigma_{n}^{2}} \sum_{i=1}^{M} \frac{K_{i}^{2}\left(x-x_{i}\right)\left(y-y_{i}\right)}{d_{i}^{2(\alpha+2)}}
\end{aligned}
$$

$$
\begin{aligned}
& J_{13}=J_{31} \\
& =E\left[\left(\frac{\partial L(R S S ; \theta)}{\partial x}\right)\left(\frac{\partial L(R S S ; \theta)}{\partial p}\right)\right] \\
& =\frac{-\alpha p}{\sigma_{n}^{4}} E\left[\sum_{i=1}^{M}\left(\frac{K_{i}\left(x-x_{i}\right)}{d_{i}^{\alpha+2}}\left(R S S_{i}-\frac{K_{i} p}{d_{i}^{\alpha}}\right)\right) \sum_{i=1}^{M}\left(\frac{K_{i}}{d_{i}^{\alpha}}\left(R S S_{i}-\frac{K_{i} p}{d_{i}^{\alpha}}\right)\right)\right] \\
& \stackrel{\substack{R S S_{i} \text { s are } \\
\text { independent }}}{=} \frac{-\alpha p}{\sigma_{n}^{4}} \sum_{i=1}^{M}\left(\frac{K_{i}^{2}\left(x-x_{i}\right)}{d_{i}^{2(\alpha+1)}} E\left[\left(R S S_{i}-\frac{K_{i} p}{d_{i}^{\alpha}}\right)^{2}\right]\right) \\
& =\frac{-\alpha p}{\sigma_{n}^{4}} \sum_{i=1}^{M} \frac{K_{i}^{2}\left(x-x_{i}\right)}{d_{i}^{2(\alpha+1)}}
\end{aligned}
$$

In a similar manner to (8) and (11),

$$
\begin{aligned}
& J_{22}=\frac{\alpha^{2} p^{2}}{\sigma_{n}^{2}} \sum_{i=1}^{M} \frac{K_{i}^{2}\left(\gamma-y_{i}\right)^{2}}{d_{i}^{2(\alpha+2)}} . \\
& J_{23}=J_{32}=\frac{-\alpha p}{\sigma_{n}^{2}} \sum_{i=1}^{M} \frac{K_{i}^{2}\left(\gamma-y_{i}\right)}{d_{i}^{2(\alpha+1)}}
\end{aligned}
$$

By substituting (8) to (13) into (6), CRLB is finally obtained as follows

$$
\begin{aligned}
& C R L B=J^{-1} \\
& =\sigma_{n}^{2}\left[\begin{array}{ccc}
\alpha^{2} p^{2} \sum_{i=1}^{M} \frac{K_{i}^{2}\left(x-x_{i}\right)^{2}}{d_{i}^{2(\alpha+2)}} & \alpha^{2} p^{2} \sum_{i=1}^{M} \frac{K_{i}^{2}\left(x-x_{i}\right)\left(y-y_{i}\right)}{d_{i}^{2(\alpha+2)}} & -\alpha p \sum_{i=1}^{M} \frac{K_{i}^{2}\left(x-x_{i}\right)}{d_{i}^{2(\alpha+1)}} \\
\alpha^{2} p^{2} \frac{K_{i=1}^{2}\left(x-x_{i}\right)\left(y-y_{i}\right)}{d_{i}^{2(\alpha+2)}} & \alpha^{2} p^{2} \sum_{i=1}^{M} \frac{K_{i}^{2}\left(\gamma-y_{i}\right)^{2}}{d_{i}^{2(\alpha+2)}} & -\alpha p \sum_{i=1}^{M} \frac{K_{i}^{2}\left(\gamma-y_{i}\right)}{d_{i}^{2(\alpha+1)}} \\
-\alpha p \sum_{i=1}^{M} \frac{K_{i}^{2}\left(x-x_{i}\right)}{d_{i}^{2(\alpha+1)}} & -\alpha p \sum_{i=1}^{M} \frac{K_{i}^{2}\left(\gamma-y_{i}\right)}{d_{i}^{2(\alpha+1)}} & \sum_{i=1}^{M} \frac{K_{i}^{2}}{d_{i}^{\alpha \alpha}}
\end{array}\right]^{-1}
\end{aligned}
$$

\section{Statistical characteristics of cognitive RSS-WLS algorithm}

Cognitive RSS-WLS algorithm [4] is an RSS-based algorithm which simultaneously estimates power and location of primary user in a CR network using WLS method. Before calculating its statistical characteristics, first we need to know how cognitive RSS-WLS algorithm works. By combining Equations (1) and (2), and neglecting the received noise power, the following equation is obtained.

$$
2 x x_{i}+2 y y_{i}+\left(\frac{K_{i}}{\mathrm{RSS}_{i}}\right)^{\frac{2}{\alpha}} \mathrm{P}-\mathrm{R}_{2}=x_{\mathrm{i}}^{2}+y_{\mathrm{i}}^{2}, i=1,2, \ldots, M .
$$

where $\rho=x^{2}+y^{2}$ and $P=(p)^{2 / \alpha}$ are auxiliary variables to linearize our model. By defining the following matrices,

$$
\mathbf{X}=\left[\begin{array}{c}
x_{1} \\
x_{2} \\
\vdots \\
x_{M}
\end{array}\right], \quad \mathbf{Y}=\left[\begin{array}{c}
y_{1} \\
y_{2} \\
\vdots \\
y_{M}
\end{array}\right], \quad \mathbf{\Lambda}=\left[\begin{array}{c}
\left(K_{1} / R S S_{1}\right)^{\frac{2}{\alpha}} \\
\left(K_{2} / R S S_{2}\right)^{\frac{2}{\alpha}} \\
\vdots \\
\left(K_{M} / R S S_{M}\right)^{\frac{2}{\alpha}}
\end{array}\right], \quad \mathbf{1}_{\mathbf{M}} \triangleq\left[\begin{array}{c}
1 \\
1 \\
\vdots \\
1
\end{array}\right]_{M \times 1},
$$

the matrix form of (15) can be written as,

$$
\mathbf{F} \varphi=\mathbf{s}
$$

where $\mathbf{F}=\left[\mathbf{2 X} \mathbf{2} \mathbf{Y} \wedge-\mathbf{1}_{\mathbf{M}}\right], \mathbf{s}=\mathbf{X} \odot \mathbf{X}+\mathbf{Y} \odot \mathbf{Y}, \phi=[x$ y $P \rho]$.

Equation (16) is a system of linear equations in matrix form where $\mathbf{F}$ is the coefficients matrix, $\phi$ is the column vector of variables, and $\mathbf{s}$ is the column vector of solutions. Although path loss exponent is naturally an unknown variable, depending on the chosen system model, it is considered to be known based on a predefined table. For example, in log-distance system model for urban area cellular radio, its value is set to 3 . As a result, path loss exponent is not taken as an unknown variable in this article. In presence of the RSS measurement error, variable vector, $\phi$, in (16) can be obtained using WLS method, which the details are discussed in [4]. In brief, in WLS method the weighted squared error due to inequality of both sides of (16) is minimized.

Since variable $\rho$ is dependent to $[x, y]$, the following auxiliary matrices are defined to separate the main variables from the dependent variable $\rho$ and also reformulate (16) to the following vector form.

$$
\mathbf{H} \triangleq\left[\begin{array}{ll}
2 \mathbf{X} 2 \mathbf{Y} \boldsymbol{\Lambda}
\end{array}\right], \quad \mathbf{v} \triangleq\left[\begin{array}{l}
x \\
y \\
P
\end{array}\right], \quad \mathbf{D} \triangleq\left[\begin{array}{lll}
1 & 0 & 0 \\
0 & 1 & 0 \\
0 & 0 & 0
\end{array}\right] .
$$

Applying the above auxiliary variables definition to (16), the error of equations, $\delta$ (i.e., difference of the sides of equations in the noisy channel) which is a zeromean random variable [6], can be obtained versus auxiliary variables as

$$
\begin{aligned}
\delta & =\left[\begin{array}{c}
\delta_{1} \\
\vdots \\
\delta_{M}
\end{array}\right] \triangleq \mathbf{F} \varphi-\mathbf{s}=2 x x_{\mathrm{i}}+2 y y_{\mathrm{i}} \\
& +\left(\frac{\mathrm{K}_{\mathrm{i}}}{\mathrm{RSS}_{\mathrm{i}}}\right)^{\frac{2}{\alpha}} P-\rho-x_{\mathrm{i}}^{2}-y_{\mathrm{i}}^{2}=\mathbf{H v}-\mathbf{1}_{\mathrm{M}} \mathbf{v}^{\mathrm{T}} \mathbf{D v}-\mathbf{s}
\end{aligned}
$$


The square error function in the WLS method is as follows [7],

$$
\mathrm{J}(\varphi) \triangle q(\mathbf{F} \varphi-\mathbf{s})^{\mathrm{T}} \mathbf{W}(\mathbf{F} \varphi-\mathbf{s})
$$

where $W$ is the weighting matrix. Denoting the covariance matrix of $\delta$, by $\Omega$, it is shown in [7] that the optimal weighting matrix which minimize $\mathrm{J}(\phi)$ is

$$
\mathbf{W}=\boldsymbol{\Omega}^{-1}=\mathbf{C}_{\delta}^{-1}=E\left[\delta \delta^{\mathbf{T}}\right]^{-1},
$$

where covariance of equations errors, $\boldsymbol{\Omega}$, is affected by the factors like error of path loss exponent estimation, shadowing factor and the input noise. By substituting the above formula for $\mathbf{W}$ and (18) into (19), the square error function is obtained as,

$$
J(v)=\left(H v-1_{M} v^{T} D v-s\right)^{T} \Omega^{-1}\left(H v-1_{M} v^{T} D v-s\right)
$$

It is shown in [8] that bias and covariance of a general optimization problem,

$$
\hat{\mathbf{v}}=\underset{\mathbf{v}}{\arg \min } J(\mathbf{v})
$$

can be approximated as,

$$
\begin{aligned}
& \operatorname{bias}(\mathbf{v}) \cong-E\left[\frac{\partial^{2} \mathrm{~J}(\mathbf{v})}{\partial \mathbf{v} \partial \mathbf{v}^{\mathbf{T}}}\right]^{-1} E\left[\frac{\partial \mathrm{J}(\mathbf{v})}{\partial \mathbf{v}}\right] \\
& \mathbf{C}_{\mathbf{v}}=E\left[\frac{\partial^{2} \mathrm{~J}(\mathbf{v})}{\partial \mathbf{v} \partial \mathbf{v}^{\mathbf{T}}}\right]^{-1} E\left[\left(\frac{\partial \mathrm{J}(\mathbf{v})}{\partial \mathbf{v}}\right)\left(\frac{\partial \mathrm{J}(\mathbf{v})}{\partial \mathbf{v}}\right)^{T}\right] E\left[\frac{\partial^{2} \mathrm{~J}(\mathbf{v})}{\partial \mathbf{v} \partial \mathbf{v}^{\mathrm{T}}}\right]^{-1}
\end{aligned}
$$

where $\mathrm{J}(v)$ is a continuous function of $v$.

The approximations in (22) and (23) are for sufficiently small noise power variances, means the variance of error of RSS measurements is small enough which is considered in this work. To derive the final relation for (22) and (23) the following relation needs to be computed for each of the expected value term used in (22) and (23).

Taking the derivative of (20) with respect to $\mathbf{v}$ leads to

$$
\begin{aligned}
& \frac{\partial J(v)}{\partial v}=2(H-1_{M} v^{T} \underbrace{\left(D^{T}+D\right)}_{2 D})^{T} \Omega^{-1}\left(H v-1_{M} v^{T} D v-s\right) \\
& =2\left(H-21_{M} v^{T} D\right)^{T} \Omega^{-1}\left(H v-1_{M} v^{T} D v-s\right)
\end{aligned}
$$

Hence the expected value of (24) is as follows.

$$
\mathrm{E}\left[\frac{\partial \mathrm{J}(\mathbf{v})}{\partial \mathbf{v}}\right]=2\left(\mathrm{H}-2 \mathbf{1}_{\mathrm{M}} \mathbf{v}^{\mathrm{T}} \mathrm{D}\right)^{\mathrm{T}} \boldsymbol{\Omega}^{-1} \mathrm{E}[\delta]=0 .
$$

Now using (24), the second expected value in (23) is

$$
\mathrm{E}\left[\left(\frac{\partial \mathrm{J}(\mathbf{v})}{\partial \mathbf{v}}\right)\left(\frac{\partial \mathrm{J}(\mathbf{v})}{\partial \mathbf{v}}\right)^{T}\right]=4\left(\mathbf{H}-2 \mathbf{1}_{\mathrm{M}} \mathbf{v}^{\mathrm{T}} \mathbf{D}\right)^{\mathrm{T}} \boldsymbol{\Omega}^{-1}\left(\mathbf{H}-\mathbf{2} \mathbf{1}_{\mathrm{M}} \mathbf{v}^{\mathrm{T}} \mathbf{D}\right) .
$$

To compute the first matrix term in (23), it needs to be partitioned to 3 column vectors as follows,

$$
E\left[\frac{\partial^{2} \mathrm{~J}(\mathbf{v})}{\partial \mathbf{v} \partial \mathbf{v}^{\mathbf{T}}}\right]=\left[\begin{array}{lll}
\mathbf{q}_{1} & \mathbf{q}_{2} & \mathbf{q}_{3}
\end{array}\right]
$$

where,

$$
\mathbf{q}_{\mathbf{1}} \triangleq E\left[\frac{\partial}{\partial x}\left(\frac{\partial J(\mathbf{v})}{\partial \mathbf{v}}\right)\right], \mathbf{q}_{2} \triangleq E\left[\frac{\partial}{\partial y}\left(\frac{\partial J(\mathbf{v})}{\partial \mathbf{v}}\right)\right], \mathbf{q}_{3} \triangleq E\left[\frac{\partial}{\partial P}\left(\frac{\partial J(\mathbf{v})}{\partial \mathbf{v}}\right)\right]
$$

Using (24), $\mathbf{q}_{1}$ can be decomposed into two additive terms as follows,

$$
\mathrm{q}_{1}=\Delta_{1}+\Delta_{2}
$$

where,

$$
\begin{aligned}
& \boldsymbol{\Delta}_{\mathbf{1}}=E\left(2 \frac{\partial}{\partial x}\left[\left(\mathbf{H}-21_{\mathbf{M}} \mathbf{v}^{\mathrm{T}} \mathbf{D}\right)^{\mathrm{T}}\right] \boldsymbol{\Omega}^{-\mathbf{1}}\left(\mathbf{H v}-\mathbf{1}_{\mathbf{M}} \mathbf{v}^{\mathrm{T}} \mathbf{D v}-\mathbf{s}\right)\right) \\
& =2 \frac{\partial}{\partial x}\left[\left(\mathbf{H}-2 \mathbf{1}_{\mathbf{M}} \mathbf{v}^{\mathrm{T}} \mathbf{D}\right)^{\mathrm{T}}\right] \boldsymbol{\Omega}^{-1} E\left[\left(\mathbf{H v}-\mathbf{1}_{\mathbf{M}} \mathbf{v}^{\mathrm{T}} \mathbf{D v}-\mathbf{s}\right)\right] \\
& =2 \frac{\partial}{\partial x}\left[\left(\mathbf{H}-2 \mathbf{1}_{\mathbf{M}} \mathbf{v}^{\mathrm{T}} \mathbf{D}\right)^{\mathrm{T}}\right] \boldsymbol{\Omega}^{-1} E[\delta]=0, \\
& \boldsymbol{\Delta}_{\mathbf{2}}=E\left(\mathbf{2}\left(\mathrm{H}-2 \mathbf{1}_{\mathrm{M}} \mathbf{v}^{\mathrm{T}} \mathbf{D}\right)^{\mathrm{T}} \boldsymbol{\Omega}^{-1} \frac{\partial}{\partial x}\left[\left(\mathbf{H v}-\mathbf{1}_{\mathrm{M}} \mathbf{v}^{\mathrm{T}} \mathbf{D v}-\mathbf{s}\right)\right]\right) \\
& =2\left(\mathbf{H}-2 \mathbf{1}_{\mathrm{M}} \mathbf{v}^{\mathrm{T}} \mathbf{D}\right)^{\mathrm{T}} \boldsymbol{\Omega}^{-1} E\left[\frac{\partial}{\partial x}\left[(\mathbf{H v}-\mathbf{1}_{\mathrm{M}} \underbrace{\mathbf{v}^{\mathrm{T}} \mathbf{D v}-\mathbf{s}}_{x^{2}+y^{2}})\right]\right] \\
& =2\left(\mathbf{H}-2 \mathbf{1}_{\mathrm{M}} \mathbf{v}^{\mathrm{T}} \mathbf{D}\right)^{\mathrm{T}} \boldsymbol{\Omega}^{-1}\left(\mathbf{H}\left[\begin{array}{lll}
1 & 0 & 0
\end{array}\right]^{\mathrm{T}}-\mathbf{2} \mathbf{1}_{\mathrm{M}} x\right)
\end{aligned}
$$

By substituting (29) and (30) into (28), $\mathbf{q}_{1}$ is as follows

$$
\mathrm{q}_{1}=2\left(\mathrm{H}-21_{\mathrm{M}} \mathbf{v}^{\mathrm{T}} \mathrm{D}\right)^{\mathrm{T}} \boldsymbol{\Omega}^{-1}\left(\mathrm{H}\left[\begin{array}{lll}
1 & 0 & 0
\end{array}\right]^{\mathrm{T}}-21_{\mathrm{M}} x\right)
$$

In a similar way used to obtain the above expression for $\mathbf{q}_{1}$, the following relations for $\mathbf{q}_{2}$ and $\mathbf{q}_{3}$ can be obtained as,

$$
\begin{aligned}
& \mathrm{q}_{2}=2\left(\mathrm{H}-21_{\mathrm{M}} \mathbf{v}^{\mathrm{T}} \mathrm{D}\right)^{\mathrm{T}} \boldsymbol{\Omega}^{-1}\left(\mathrm{H}\left[\begin{array}{lll}
0 & 1 & 0
\end{array}\right]^{\mathrm{T}}-21_{\mathrm{M} \gamma}\right) \\
& \mathbf{q}_{3}=2\left(\mathbf{H}-2 \mathbf{1}_{\mathrm{M}} \mathbf{v}^{\mathrm{T}} \mathbf{D}\right)^{\mathrm{T}} \boldsymbol{\Omega}^{-1}\left(\mathbf{H}\left[\begin{array}{lll}
0 & 0 & 1
\end{array}\right]^{\mathrm{T}}\right)
\end{aligned}
$$

By substituting (31), (32), and (33) into (27), the expected value of the second derivative of (20) with respect to $\mathbf{v}$ is obtained as,

$$
\begin{aligned}
& E\left[\frac{\partial^{2} \mathrm{~J}(\mathbf{v})}{\partial \mathbf{v} \mathrm{v}^{\mathrm{T}}}\right]=\mathbf{2}\left(\mathbf{H}-\mathbf{2} \mathbf{1}_{\mathrm{M}} \mathbf{v}^{\mathrm{T}} \mathbf{D}\right)^{\mathrm{T}} \boldsymbol{\Omega}^{-1}\left(\mathbf{H}\left(\begin{array}{lll}
1 & 0 & 0 \\
0 & 1 & 0 \\
0 & 0 & 1
\end{array}\right)-\left(2 \mathbf{1}_{\mathrm{M}} x+2 \mathbf{1}_{\mathrm{M}} \gamma\right)\right) \\
& =2\left(\mathrm{H}-21_{\mathrm{M}} v^{\mathrm{T}} \mathrm{D}\right)^{\mathrm{T}} \boldsymbol{\Omega}^{-1}\left(\mathrm{H}-21_{\mathrm{M}} v^{\mathrm{T}} \mathrm{D}\right)
\end{aligned}
$$

By substituting (25) and (34) into (22), since $\delta$ is a zero-mean random variable [6], the bias of estimation error is obtained as, 


$$
\operatorname{bias}(\mathbf{v})=-E\left[\frac{\partial^{2} J(\mathbf{v})}{\partial \mathbf{v} \partial \mathbf{v}^{\mathrm{T}}}\right]^{-1}\left(2\left(\mathbf{H}-2 \mathbf{1}_{\mathrm{M}} \mathbf{v}^{\mathrm{T}} \mathbf{D}\right)^{\mathrm{T}} \boldsymbol{\Omega}^{-1} E[\delta]\right)=0
$$

By the above, it can be concluded that RSS-WLS algorithm is an unbiased location and power estimation algorithm. Finally, by substituting (26) and (34) into (23), the closed form of the 3 by 3 covariance matrix of estimation error is obtained as,

$$
C_{V}=\left(\left(H-21_{M} v^{T} D\right)^{T} \Omega^{-1}\left(H-21_{M} v^{T} D\right)\right)^{-1}
$$

where the first two rows and columns of $\mathbf{C}_{\mathbf{v}}$ consist of covariance matrix of positioning error, while the last diagonal element is the covariance of power estimation error. Since cognitive RSS-WLS estimator is unbiased, covariance matrix of estimation error of (36) is also covariance matrix of the estimator. It should be noted that since WLS is a generalization of LS method, the error statistics of RSS-LS method for the same scenario can be obtained by substituting the weighting matrix $\mathbf{W}$ with the identity matrix in (23) and (24).

\section{Comparison}

Based on [4], in an AWGN channel, optimal weighting matrix, $\boldsymbol{\Omega}^{-1}$, is a diagonal matrix with diagonal elements of

$$
\left(\mathbf{W}_{o p t}\right)_{i i}=\left(\boldsymbol{\Omega}^{-1}\right)_{i i}=\frac{\alpha^{2} K_{i}^{2} p^{2}}{4 \sigma_{n}^{2} d_{i}^{2(\alpha+2)}}
$$

By substituting (17) and (37) into (36) and using (1) and some basic calculations, covariance matrix of cognitive RSS-WLS estimator in terms of unknown variables, $x, y$ and $P$, is obtained as follows,

$C_{v}$

$$
=\sigma_{n}^{2}\left[\begin{array}{ccc}
\alpha^{2} p^{2} \sum_{i=1}^{M} \frac{K_{i}^{2}\left(x-x_{i}\right)^{2}}{d_{i}^{2(\alpha+2)}} & \alpha^{2} p^{2} \sum_{i=1}^{M} \frac{K_{i}^{2}\left(x-x_{i}\right)\left(y-\gamma_{i}\right)}{d_{i}^{2(\alpha+2)}}-\alpha p \sum_{i=1}^{M} \frac{K_{i}^{2}\left(x-x_{i}\right)}{d_{i}^{2(\alpha+1)}} \\
\alpha^{2} p^{2} \sum_{i=1}^{M} \frac{K_{i}^{2}\left(x-x_{i}\right)\left(y-\gamma_{i}\right)}{d_{i}^{2(\alpha+2)}} \alpha^{2} p^{2} \sum_{i=1}^{M} \frac{K_{i}^{2}\left(\gamma-\gamma_{i}\right)^{2}}{d_{i}^{2(\alpha+2)}} & -\alpha p \sum_{i=1}^{M} \frac{K_{i}^{2}\left(y-\gamma_{i}\right)}{d_{i}^{2(\alpha+1)}} \\
-\alpha p \sum_{i=1}^{M} \frac{K_{i}^{2}\left(x-x_{i}\right)}{d_{i}^{2(\alpha+1)}} & -\alpha p \sum_{i=1}^{M} \frac{K_{i}^{2}\left(\gamma-\gamma_{i}\right)}{d_{i}^{2(\alpha+1)}} & \sum_{i=1}^{M} \frac{K_{i}^{2}}{d_{i}^{\alpha}}
\end{array}\right]^{-1}
$$

Comparing (14) and (38) shows that covariance of location estimation using cognitive RSS-WLS method is equal to CRLB. since we have obtained this result by assuming the input noise to be sufficiently small, we can say that cognitive RSS-WLS estimator is asymptotically efficient.

\section{Conclusion}

To conclude, cognitive RSS-WLS algorithm can be used to simultaneously estimate location and power of the primary user in CR system in an AWGN channel.
Through this article, first CRLB of RSS-based algorithms for CR setup in an AWGN channel is obtained, then the closed form expression for mean and covariance of estimation error of cognitive RSS-WLS algorithm is calculated. At the end, based on the results of the previous sections, an analytical performance evaluation of cognitive RSS-WLS algorithm is done and it is analytically shown that cognitive RSS-WLS estimator is asymptotically efficient.

\section{Authors' contributions}

In this article, we followed three goals. First, we calculated CRLB of RSSbased algorithms for CR setup in an AWGN channel. Second, we obtained the closed form expression for mean and covariance of estimation error of cognitive RSS-WLS algorithm. Third, we analytically showed that cognitive RSS-WLS estimator is asymptotically efficient in an AWGN channel. All authors read and approved the final manuscript.

\section{Competing interests}

The authors are with Spread Spectrum Lab of Faculty of Electrical and Computer Engineering, Khaje Nasir Toosi University of Technology, Tehran, Iran.

Received: 17 May 2011 Accepted: 5 February 2012

Published: 5 February 2012

\section{References}

1. J Mitola III, GQ Maguire, Cognitive radio: Making software radios more personal. IEEE Personal Commun Mag. 64, 13-18 (1999)

2. KW Cheung, HC So, WK Ma, YT Chan, Received signal strength based mobile positioning via constrained weighted least squares, in Proceedings of the IEEE International Conference on Acoustic, Speech and Signal Processing (ICASSP '03), vol. 5. Hong Kong, pp. 137-140 (April 2003)

3. S Kim, $\mathrm{H}$ Jeon, J Ma, Robust localization with unknown transmission power for cognitive radio, in Proc IEEE Int Conf on Military Communications (October 2007)

4. M Kazemi, M Ardebilipour, N Nouri, Improved weighted RSS positioning algorithm for cognitive radio, in International Conference on Signal Processing (ICSP'10) (2010)

5. A Papoulis, SU Pillai, Probability, Random Variables and Stochastic Processes, 4th edn. (McGraw Hill, New York, 2002)

6. KW Cheung, HC So, W-K Ma, YT Chan, A constrained least squares approach to mobile positioning: algorithms and optimality. EURASIP Journal on Applied Signal Processing. 2006 (2006). Article ID 20858, 23

7. AC Aitken, On least squares and linear combinations of observations. Proc Royal Soc Edinburgh. 55, 42-48 (1935)

8. KW Cheung, HC So, A multi-dimensional scaling framework for mobile location using time-of-arrival measurements. IEEE Trans Signal Process. 53(2), 460-470 (2005)

doi:10.1186/1687-1499-2012-31

Cite this article as: Kazemi et al:: Statistical analysis of linear spatial holes estimators in cognitive radio. EURASIP Journal on Wireless Communications and Networking 2012 2012:31. 